\title{
lodine intake and status of UK women of childbearing age recruited at the University of Surrey in the winter
}

\author{
Sarah C. Bath, Michelle L. Sleeth, Marianne McKenna, Alan Walter, Andrew Taylor and \\ Margaret P. Rayman* \\ Department of Nutritional Sciences, Faculty of Health and Medical Sciences, University of Surrey, Guildford, \\ Surrey GU2 $7 X H, U K$
}

(Submitted 28 March 2014 - Final revision received 14 July 2014 - Accepted 6 August 2014 - First published online 2 October 2014)

\section{Abstract}

As intra-thyroidal iodine stores should be maximised before conception to facilitate the increased thyroid hormone production during pregnancy, women who are planning to become pregnant should ideally consume $150 \mu \mathrm{g}$ iodine/d (US RDA). As few UK data exist for this population group, a cross-sectional study was carried out at the University of Surrey to assess the iodine intake and status of women of childbearing age. Total iodine excretion was measured from $24 \mathrm{~h}$ urine samples in fifty-seven women; iodine intake was estimated by assuming that $90 \%$ of ingested iodine was excreted. The average iodine intake was also estimated from $48 \mathrm{~h}$ food diaries that the participants completed. The median urinary iodine concentration value $(63.1 \mu \mathrm{g} / \mathrm{l})$ indicated the group to be mildly iodine deficient by WHO criteria. By contrast, the median $24 \mathrm{~h}$ urinary iodine excretion value $(149.8 \mu \mathrm{g} / 24 \mathrm{~h})$ indicated a relatively low risk of iodine deficiency. The median estimated iodine intake, extrapolated from urinary excretion, was $167 \mu \mathrm{g} / \mathrm{d}$, whereas it was lower, at $123 \mu \mathrm{g} / \mathrm{d}$, when estimated from the $48 \mathrm{~h}$ food diaries. Iodine intake estimated from the food diaries and $24 \mathrm{~h}$ urinary iodine excretion were strongly correlated $(r 0 \cdot 75, P<0 \cdot 001)$. The intake of milk, eggs and dairy products was positively associated with iodine status. The iodine status of this UK cohort is probably a best-case scenario as the women were mostly nutrition students and were recruited in the winter when milkiodine content is at its highest; further study in more representative cohorts of UK women is required. The present study highlights the need for revised cut-off values for iodine deficiency that are method- and age group-specific.

\section{Key words: Iodine: Iodine deficiency: Milk: UK: Public health: Pregnancy: Diets}

Iodine is required for the production of thyroid hormones (thyroxine and tri-iodothyronine), which in turn are required for normal fetal brain and neurological development ${ }^{(1)}$. A sufficient intake of iodine during pregnancy is needed to prevent the potential adverse effects of deficiency on the developing brain that can persist throughout life. Severe iodine deficiency during pregnancy is well known to result in cretinism, a disorder associated with mental retardation, deafness and motor dysfunction in children. Even mild-to-moderate iodine deficiency is associated with lower intelligent quotient and reading $^{(2)}$ and spelling ability ${ }^{(3)}$ up to the age of 9 years.

Although it is important for pregnant women to have a sufficient intake of iodine, it is arguably more important that women of childbearing age, particularly those planning a pregnancy, should consume enough; emerging data suggest that pregnant women who have had a regular adequate intake of iodine have a better thyroid hormone profile than those who only begin iodine supplementation when they become pregnant ${ }^{(4-6)}$. This is probably because the thyroid can store iodine that can be drawn on during the course of pregnancy ${ }^{(5)}$. As many pregnancies are unplanned and because pregnancy may not be confirmed until several weeks into the first trimester - a critical period for thyroid hormone need - it is essential that women of childbearing age consume an adequate amount of iodine on a regular basis and meet the RDA (US RDA) of $150 \mu \mathrm{g} / \mathrm{d}^{(7)}$.

For many years, the UK has been considered to be an iodine-sufficient country, despite reports of endemic goitre in the past ${ }^{(8)}$. Iodine deficiency was eradicated in the UK through changes in the dairy-farming industry in the $1930 \mathrm{~s}$ (i.e. through increased use of iodine-fortified cattle feed and iodine-containing disinfectants) and concurrent increase in milk consumption in the post-war years ${ }^{(8)}$; from the 1960 s, iodine sufficiency was assumed in the UK and there was a dearth of data on the status of the population. In fact, at the time that the present study was conducted, there were no national UK data on the iodine status of the population ${ }^{(9)}$ and just two studies ${ }^{(10,11)}$ on the iodine status of pregnant women. There are now UK-wide data that suggest that teenage schoolgirls are mildly iodine deficient ${ }^{(12)}$ and regional studies

Abbreviations: EAR, estimated average requirement; UIC, urinary iodine concentration.

*Corresponding author: Professor M. P. Rayman, fax + 441483 686401, email m.rayman@surrey.ac.uk 
that report iodine deficiency in UK pregnant women ${ }^{(2,10,13,14)}$. However, data on the iodine status of UK women of childbearing age are still lacking, and few studies have assessed measures of both dietary iodine intake and status. Assessment of urinary iodine excretion is a widely accepted method for measuring iodine status; approximately $90 \%$ of ingested iodine is ultimately excreted in the urine ${ }^{(1)}$. Total iodine excretion in a $24 \mathrm{~h}$ urine collection is considered to be preferable to iodine concentration measured in spot-urine samples for assessing iodine status in an individual $^{(15,16)}$.

In the present study, we assessed iodine status from $24 \mathrm{~h}$ urine collections in UK women of childbearing age, i.e. in a cohort of women who could potentially become pregnant in the short-to-medium term. In addition, through the use of food diaries, we investigated which iodine-rich food groups had the most influence on iodine status. We also compared two methods for estimating iodine intake: (1) extrapolation from $24 \mathrm{~h}$ urinary iodine excretion and (2) estimation of intake by dietary assessment. This is the first study to be conducted on the iodine status of women of childbearing age in the UK using these methods and the first to report the comparison of two methods for estimating iodine intake.

\section{Experimental methods}

\section{Recruitment and protocol}

The study was conducted at the University of Surrey, Guildford, UK. Women of childbearing age (defined as still menstruating) were recruited during January and February 2007 and again in January and February 2008. Women were recruited by word of mouth in the University through friends and colleagues and through responses to an email advertisement. Exclusion criteria included current or recent pregnancy (in the last 6 months), breast-feeding, known thyroid disease and use of medication for thyroid disease - thyroxine, amiodarone, carbimazole or propylthiouracil.

The WHO recommends that the median iodine concentration estimated from spot-urine samples be compared with the published cut-off values for adequacy for assessing the iodine status of a population ${ }^{(17)}$. However, urinary iodine concentration (UIC) is not suitable for the assessment of individual iodine status; for this purpose, multiple spot-urine samples or $24 \mathrm{~h}$ urine collections (for the measurement of total iodine excretion) are required ${ }^{(18)}$. Though the $24 \mathrm{~h}$ iodine excretion estimated from a single $24 \mathrm{~h}$ urine collection does not account for the day-to-day variability in iodine intake, it does overcome the variability associated with urine volume that affects the interpretation of iodine concentration in a spot-urine sample and for that reason can be considered preferable to a single spot-urine sample ${ }^{(15,16)}$. The participants who volunteered to take part in the study were required to collect all the urine passed in a $24 \mathrm{~h}$ time period (completeness of the sample was self-reported). The participants were provided with clear instructions, a wide-necked jug and a leak-proof 5-litre container (both had been acid-washed and rinsed before use). They were advised not to wash the jug between urine collections and to ensure that the container lid was well sealed. The total urine volume of each participant was measured; $20 \mathrm{ml}$ aliquots were taken and stored at $-20^{\circ} \mathrm{C}$ until analysis.

The participants completed a questionnaire to provide basic information on date of birth, use of nutritional supplements or medication and any dietary exclusion practised. They were required to keep a detailed food diary both for the $24 \mathrm{~h}$ before urine collection and during the $24 \mathrm{~h}$ of urine collection.

The study was conducted according to the guidelines laid down in the Declaration of Helsinki, and all procedures involving human subjects were approved by the University of Surrey Ethics Committee. Written informed consent was obtained from all participants.

\section{Determination of urinary iodine concentration}

Iodine concentration was measured on a Thermo Elemental X-Series ICP-MS system (Thermo Fisher Scientific) in the Trace Element Laboratory at the University of Surrey. To produce samples in the analytical range for inductively coupled plasma MS, the samples were first diluted with an alkaline diluent. The diluent used was prepared by dissolving $3.32 \mathrm{~g}$ of $\mathrm{NH}_{4} \mathrm{H}_{2} \mathrm{PO}_{4}$ and $1 \cdot 16 \mathrm{~g}$ of $\left(\mathrm{NH}_{4}\right)_{2} \mathrm{H}_{2}$ EDTA (Analar grade; SigmaAldrich) in deionised water, adding $10.0 \mathrm{ml}$ of $\mathrm{NH}_{3}$ solution (specific gravity 0.88), and making up to $1000 \mathrm{ml}$ with deionised water. The final diluent solution contained $0 \cdot 14 \mathrm{M}-$ $\mathrm{NH}_{3}, 0.003 \mathrm{M}-\left(\mathrm{NH}_{4}\right)_{2} \mathrm{H}_{2}$ EDTA and $0.029 \mathrm{M}-\mathrm{NH}_{4} \mathrm{H}_{2} \mathrm{PO}_{4}$. Using the alkaline diluent, $400 \mu \mathrm{l}$ of each participant's urine sample were made up to $10 \mathrm{ml}$. Standard iodine solutions were prepared with KI (Analar grade; Fisher Scientific) for the construction of calibration curves. To obtain matrix-matched standards, $400 \mu \mathrm{l}$ of control urine (taken from laboratory stock with median UIC of 80.6 and $46.5 \mu \mathrm{g} / 1$ in 2007 and 2008, respectively) were added to $400 \mu \mathrm{l}$ of each standard. An internal standard was added to all samples: rhodium $\left({ }^{103} \mathrm{Rh}\right)$ and iridium $\left({ }^{192} \mathrm{Ir}\right)$ (both obtained from SPEX CertiPrep Limited) were made up in a working standard solution of $1 \mathrm{mg} / \mathrm{l}$ (parts per million) in $1 \% \mathrm{v} / \mathrm{v}$ $\mathrm{HNO}_{3}$ (Trace analysis grade; Fisher Scientific). This was made up 1:10 with the diluent and $150 \mu$ l was added to each tube.

To evaluate the accuracy of the method, a number of certified reference materials were used (Seronorm urine (Bio-Stat House) in 2007 and EQUIP (ensuring the quality of urinary iodine procedures) ${ }^{(19)}$ samples in 2008). The mean values obtained for the EQUIP-certified reference materials (U02, U05, U09, U10) were as follows: 28.9 (SD 0.1) $\mu \mathrm{g} / \mathrm{l}$ ( $n$ 2) for U02 (certified mean $28.7 \mu \mathrm{g} / \mathrm{l}$, range $20 \cdot 1-37 \cdot 3 \mu \mathrm{g} / \mathrm{l}$ ); 45.9 (SD 0.0$) \mu \mathrm{g} / 1$ ( $n$ 2) for U05 (certified mean $45.0 \mu \mathrm{g} / \mathrm{l}$, range 31.5-58.5 $\mathrm{g} / \mathrm{l}$ ); $298.0(\mathrm{SD} 0.0) \mu \mathrm{g} / \mathrm{l}(n$ 2) for U09 (certified mean $296.3 \mu \mathrm{g} / \mathrm{l}$, range $251.9-340.7 \mu \mathrm{g} / \mathrm{l}$ ); 11.4 (SD 0.6) $\mu \mathrm{g} / 1$ ( $n$ 2) for U10 (certified mean $12 \cdot 2 \mu \mathrm{g} / \mathrm{l}$, range $8.5-15.9 \mu \mathrm{g} / \mathrm{l}$ ) and 264 (SD $1 \cdot 0) \mu \mathrm{g} / \mathrm{l}(n$ 3) for Seronorm urine (certified mean $282 \mu \mathrm{g} / \mathrm{l}$, range $264-300 \mu \mathrm{g} / \mathrm{l})$.

\section{Derivation of total urinary iodine excretion and extrapolation to give estimated iodine intake}

Total $24 \mathrm{~h}$ urinary iodine excretion was calculated for each participant by multiplying iodine concentration $(\mu \mathrm{g} / \mathrm{l})$ by total urine volume (in litres) collected. It has been estimated 
that approximately $90 \%{ }^{(1)}$ of ingested iodine is eventually excreted in the urine, and this assumption allows dietary iodine intake to be estimated by dividing total urinary iodine excretion $(\mu \mathrm{g} / 24 \mathrm{~h})$ by $0 \cdot 90$.

\section{Analysis of dietary iodine intake}

The information recorded in the $48 \mathrm{~h}$ food diaries was entered into the WinDiets Research programme (version 2005; Robert Gordon University) to estimate iodine intake both on the day before and on the day of urine collection. Where portion weights were not recorded in the diaries, medium portion sizes were entered ${ }^{(20)}$. The iodine content of reported multivitamin and mineral supplements was added to the total iodine intake estimated from the food diaries on each day and an average iodine intake across the $48 \mathrm{~h}$ was calculated. The quantity of iodine-rich foods (milk, meat, fish and eggs) and soya drinks (as a potential replacement for iodine-rich cows' milk) consumed was extracted from the participants' diaries from each $24 \mathrm{~h}$ period and an average was calculated; the average value for each food item was then used to examine the relationship with urinary iodine excretion.

\section{Classification of iodine status and estimation of the prevalence of iodine deficiency}

The median UIC of the study group was compared with the WHO criteria for the risk of iodine deficiency (Table 1). To calculate the risk in individuals, total urinary iodine excretion in $24 \mathrm{~h}$ was compared with values reported in other studies ${ }^{(15,21)}$ and with the thresholds (i.e. $\mu \mathrm{g} / \mathrm{d}$ ) proposed in populationbased studies (Table 1$)^{(22)}$.

The Dietary Reference Intake values published by the Institute of Medicine ${ }^{(7)}$ were used for evaluating iodine intake (either extrapolated from urinary iodine excretion or estimated from food diaries); neither the UK Dietary Reference Values ${ }^{(23)}$ nor the WHO recommendations ${ }^{(17)}$ provide a value for the estimated average requirement (EAR), which is required for prevalence estimates of nutrient deficiencies in a population ${ }^{(24)}$. The percentage of women with an iodine intake below the adult EAR $(95 \mu \mathrm{g} / \mathrm{d}){ }^{(7)}$ was used to describe the prevalence of deficiency in the cohort.

Table 1. Classification of the risk of iodine deficiency using measures of urinary iodine concentration and total iodine excretion in a $24 \mathrm{~h}$ period

\begin{tabular}{lcc}
\hline $\begin{array}{l}\text { Risk of iodine } \\
\text { deficiency }\end{array}$ & $\begin{array}{c}\text { Urinary iodine } \\
\text { concentration }\left(\mu \mathrm{g} / \mathrm{l}^{*}\right.\end{array}$ & $\begin{array}{c}24 \mathrm{~h} \text { urinary iodine } \\
\text { excretion }(\mu \mathrm{g} / \mathrm{d}) \dagger\end{array}$ \\
\hline None & $100-199$ & $\geq 100$ \\
Mild & $50-99$ & $\geq 50$ and $<100$ \\
Moderate & $20-49$ & $\geq 25$ and $<50$ \\
Severe & $<20$ & $<25$ \\
\hline
\end{tabular}

${ }^{*}$ WHO criteria for adult populations ${ }^{(17)}$.

†Criteria for iodine deficiency in individuals for $24 \mathrm{~h}$ urinary iodine excretion derived from Thomson et al. ${ }^{(15)}$ and Als et al. ${ }^{(21)}$

\section{Statistical analysis}

As variables were not normally distributed, median and interquartile ranges are reported; variables were transformed using the natural logarithm to allow parametric testing where possible. Data on the intake of food groups (e.g. fish and milk) were not normally distributed, even after transformation, and therefore non-parametric tests were used.

Independent $t$ tests or one-way ANOVA were used to compare (log-transformed) $24 \mathrm{~h}$ urinary iodine excretion values between the groups. A paired $t$ test was used to compare intake over the $48 \mathrm{~h}$ of dietary records and the two methods for iodine intake estimation. Analysis of the correlation between two continuous variables was conducted using Pearson's correlation when both variables were normally distributed or Spearman's rank when variables were not normally distributed. Forward stepwise linear regression (using log-transformed $24 \mathrm{~h}$ urinary iodine excretion values) was performed to evaluate the most important dietary predictors of iodine status; all dietary variables and dose of iodine in a multivitamin and mineral supplement were entered as independent variables.

Bland-Altman plots were used to compare the two methods for iodine intake estimation (i.e. extrapolation from $24 \mathrm{~h}$ urinary iodine excretion or from estimation from food diaries). The mean difference between the two methods was plotted against the mean of the methods.

Statistical significance was set at $P<0 \cdot 05$, and analysis was performed with the Statistical Package for Social Sciences (version 21.0; SPSS, Inc.).

\section{Results}

A total of twenty-six women volunteered to participate in 2007 and thirty-one in 2008, giving a total of fifty-seven women of childbearing age. Approximately $90 \%$ of the participants were studying for a degree in nutrition or nutrition/dietetics. The median age of the participants was 23 (range 19-45) years. Among these participants, five ( $8.8 \%)$ were lacto-ovo vegetarians and three $(5.3 \%)$ were pescatarians (excluded meat and poultry but included fish); there were no vegans in the study.

\section{lodine excretion and estimated iodine intake}

A summary of UIC, $24 \mathrm{~h}$ urinary iodine excretion and estimated iodine intake (extrapolated from urinary iodine excretion) is given in Table 2 . The median UIC value $(63.1 \mu \mathrm{g} / \mathrm{l})$ and the fact that $31.6 \%$ ( $n$ 18) of the participants had a UIC $<50 \mu \mathrm{g} / \mathrm{l}$ indicated the group to be mildly iodine deficient by the WHO criteria $^{(17)}$. However, the median $24 \mathrm{~h}$ urinary iodine excretion value $(149 \cdot 8 \mu \mathrm{g} / 24 \mathrm{~h})$ indicated the same group to have an adequate iodine status according to the criteria listed in Table $1^{(15,21)}$

When $24 \mathrm{~h}$ urinary iodine excretion was extrapolated to estimate daily intake (on the basis that $90 \%$ is excreted), the median $(167 \mu \mathrm{g} / \mathrm{d})$ was above the adult RDA of $150 \mu \mathrm{g} / \mathrm{d}^{(17)}$. The estimated iodine intake for the fifty-seven participants is shown in Fig. 1; the dotted lines denote the RDA and EAR 
Table 2. Summary of urinary iodine concentration, $24 \mathrm{~h}$ urine volume, $24 \mathrm{~h}$ urinary iodine excretion and extrapolated daily iodine intake for all the fifty-seven participants

(Median values and 25th-75th percentiles)

\begin{tabular}{lcc}
\hline & Median & 25th-75th percentiles \\
\hline Urinary iodine concentration $(\mu \mathrm{g} / \mathrm{l})$ & 63.1 & $40 \cdot 8-95 \cdot 0$ \\
Urine volume (litres/24 $\mathrm{h})$ & 2.52 & $1 \cdot 76-3.02$ \\
Total iodine excretion $(\mu \mathrm{g} / 24 \mathrm{~h})$ & 149.8 & $102 \cdot 4-220.5$ \\
lodine intake $(\mu \mathrm{g} / \mathrm{d})^{\star}$ & 167 & $114-245$ \\
\hline
\end{tabular}

*Estimated by extrapolation from urinary excretion (dividing by 0.90 ).

for adults ${ }^{(7)}$ and the EAR for pregnant women ${ }^{(7)}$. Among the participants, $14 \%$ ( $n$ 8) had an estimated intake below the adult EAR of $95 \mu \mathrm{g} / \mathrm{d}^{(7)}$ and $40 \cdot 3 \%$ ( $n$ 23) had an estimated iodine intake below the adult RDA $(150 \mu \mathrm{g} / \mathrm{d})$. Furthermore, $42 \%(n 24)$ had an iodine intake below the EAR for pregnancy $(160 \mu \mathrm{g} / \mathrm{d})^{(7)}$, none of whom was taking a multivitamin and mineral supplement containing iodine.

The median iodine intake estimated from the food diaries was above the adult EAR $(95 \mu \mathrm{g} / \mathrm{d})$, but below the RDA $(150 \mu \mathrm{g} / \mathrm{d})$ for both $24 \mathrm{~h}$ periods of dietary records as was the average (Table 3). A total of sixteen participants (28.1\%) had an iodine intake (averaged over the two $24 \mathrm{~h}$ periods) that was below the EAR, a value higher than that estimated from the extrapolation of $24 \mathrm{~h}$ urinary iodine excretion ( $n$ 8, 14\%). A total of thirty-four participants (59.6\%) had an average iodine intake below the EAR for pregnancy.

The values estimated from the $48 \mathrm{~h}$ food diaries showed that there was no significant difference in iodine intake between the two $24 \mathrm{~h}$ periods (paired $t$ test: $P=0 \cdot 23$ ). This suggests little variation in iodine intake over two consecutive days in this population.

\section{Relationship between the two methods for estimating iodine intake}

Iodine intake estimated from the food diaries was strongly correlated with $24 \mathrm{~h}$ urinary iodine excretion (Table 3; Fig. 2(a)). Fig. 2 shows the correlation and agreement between the two methods for estimating iodine intake - i.e., estimation from the $48 \mathrm{~h}$ food diaries and supplements or from extrapolation of the $24 \mathrm{~h}$ urinary iodine excretion. There was a significant difference in the iodine intake estimated using the two methods (paired $t$ test: $P=0 \cdot 001$ ). The Bland-Altman plot showed that there was a considerable lack of agreement between the methods as, on average, iodine intake estimated from the food diaries was lower than that estimated from urinary excretion (mean difference $-18.8 \mu \mathrm{g} / \mathrm{d}$ ) and, on an individual basis, the difference ranged from -144.4 to $106.8 \mu \mathrm{g} / \mathrm{d}$ (Fig. 2(b)); this suggests that the methods cannot be used interchangeably.

\section{Dietary exclusions, use of iodine-containing supplements and intake of iodine-rich food items}

There was no significant difference in the $24 \mathrm{~h}$ urinary iodine excretion values among omnivores, vegetarians and pescatarians $(P=0 \cdot 17)$. Use of iodine-containing multivitamin and

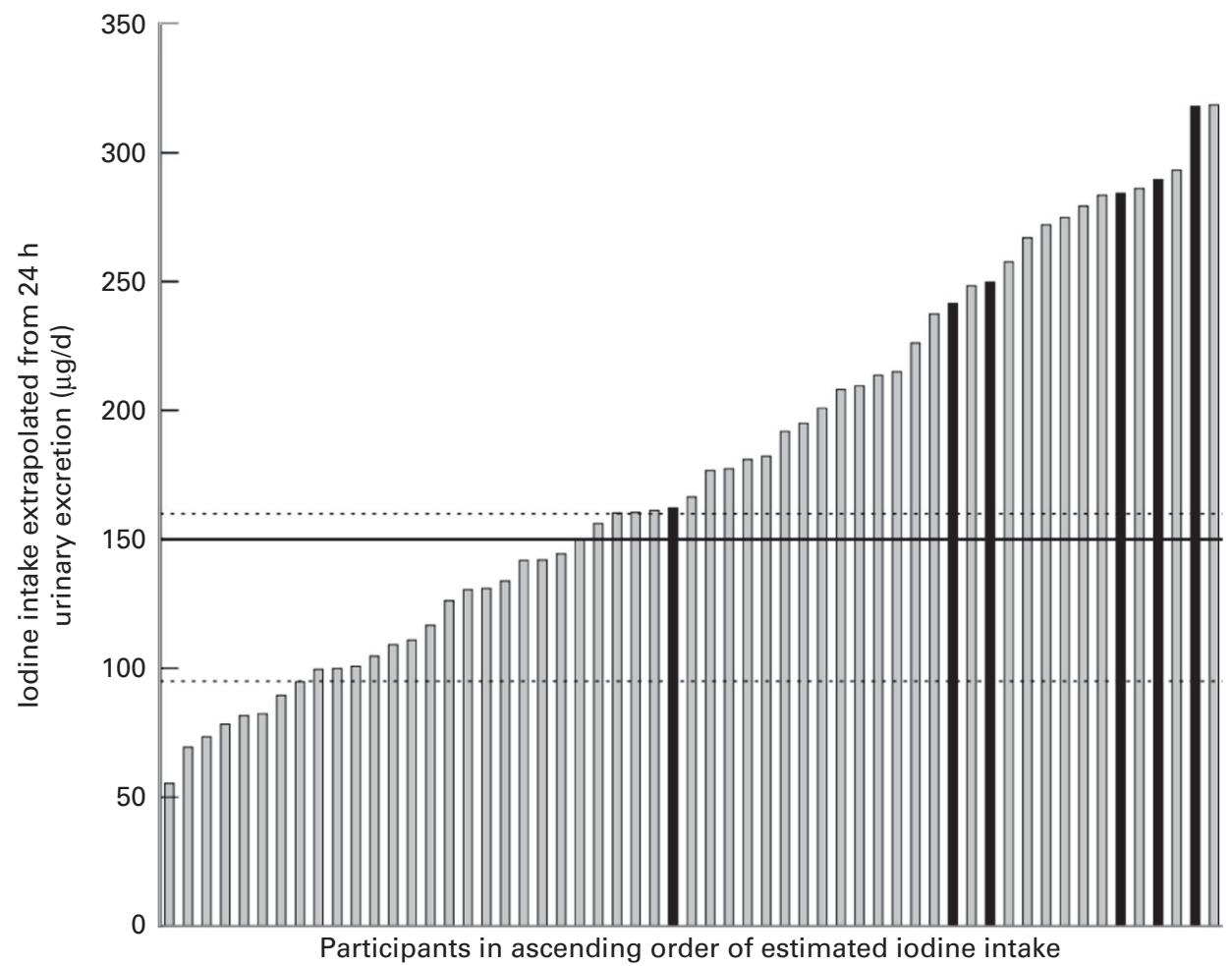

Fig. 1. Participants in ascending order of iodine intake extrapolated from $24 \mathrm{~h}$ urinary iodine excretion. $\mathbf{\square}$ represents participants who took a supplement containing iodine. - (middle) represents the RDA for adults $(150 \mu \mathrm{g} / \mathrm{d})^{(7)}$. - - - represents the Institute of Medicine's estimated average requirement (EAR) values: - - $($ lower) represents the EAR for adults $(95 \mu \mathrm{g} / \mathrm{d})$ and - - (upper) represents the EAR for pregnant women $(160 \mu \mathrm{g} / \mathrm{d})^{(7)}$. 
Table 3. lodine intake estimated from food diaries plus supplements (Median values and 25th-75th percentiles)

\begin{tabular}{|c|c|c|c|c|}
\hline \multirow[b]{2}{*}{ lodine intake $(\mu \mathrm{g} / \mathrm{d})$} & \multirow[b]{2}{*}{ Median } & \multirow{2}{*}{$\begin{array}{l}\text { 25th-75th } \\
\text { percentiles }\end{array}$} & \multicolumn{2}{|c|}{$\begin{array}{l}\text { Correlation with } \\
24 \mathrm{~h} \text { urinary iod- } \\
\text { ine excretion } \\
(\mu \mathrm{g} / 24 \mathrm{~h})\end{array}$} \\
\hline & & & $r$ & $P$ \\
\hline $\begin{array}{l}24 \mathrm{~h} \text { before urine } \\
\text { collection }\end{array}$ & 148 & $82-228$ & $0 \cdot 70$ & $<0.0001$ \\
\hline $\begin{array}{l}\text { During } 24 \mathrm{~h} \text { of } \\
\text { urine collection }\end{array}$ & 116 & $74-213$ & 0.66 & $<0.0001$ \\
\hline $\begin{array}{l}\text { Average of the two } \\
24 \mathrm{~h} \text { dietary records }\end{array}$ & 123 & $87-211$ & 0.75 & $<0.0001$ \\
\hline
\end{tabular}

mineral supplements in which the dose of iodine ranged from 75 to $200 \mu \mathrm{g} / \mathrm{d}$ was reported by six participants (10.5\%). Participants who used an iodine-containing supplement excreted significantly more iodine in $24 \mathrm{~h}$ than non-supplement users ( $240 v$. $144 \mu \mathrm{g} / 24 \mathrm{~h} ; P=0.01)$.

In the $24 \mathrm{~h}$ before urine collection and/or during the $24 \mathrm{~h}$ of urine collection, cows' milk was consumed by $84.3 \%$ of the participants ( $n$ 48), fish was consumed by $28 \cdot 1 \%$ ( $n$ 16) and eggs by $21 \cdot 1 \%$ ( $n$ 12). Soya drinks were consumed by five participants ( $8.8 \%)$, and there was a negative correlation between the intake of soya drinks and that of cows' milk $(r-0.43$, $P=0.001)$, suggesting that the participants were using soya drinks as an alternative to cows' milk.

The intake of milk, dairy products, fish and eggs was positively correlated with $24 \mathrm{~h}$ urinary iodine excretion, whereas the intake of soya drinks and meat was negatively correlated (Table 4). The strongest correlation was observed for milk, followed by eggs (Table 4). When the variables listed in Table 4 were entered into a linear regression model along with the dose of iodine in any supplement used (with log-transformed $24 \mathrm{~h}$ urinary iodine excretion as the dependent variable), milk intake $(P<0 \cdot 0005)$, egg consumption $(P=0.004)$ and intake of other dairy products $(P=0.013)$ were all found to be significant predictors of iodine status; the intake of soya drinks, fish and meat was not a significant predictor in the final model, which explained $49.3 \%$ of the variation in $24 \mathrm{~h}$ urinary iodine excretion $\left(r^{2} 0 \cdot 493\right)$.

\section{Discussion}

\section{lodine intake and status}

The median UIC $(63.1 \mu \mathrm{g} / \mathrm{l})$ is suggestive of mild iodine deficiency when using the current WHO cut-off values for adequacy $^{(17)}$, echoing the finding of mild iodine deficiency in UK schoolgirls ${ }^{(12)}$. However, if using the more recently proposed cut-off values of $60-70 \mu \mathrm{g} / 1$ for adults ${ }^{(22)}$, these women would be classified as having an adequate iodine status. Indeed, based on the $24 \mathrm{~h}$ urinary iodine excretion, the risk of deficiency within the group was low, i.e. after accounting for total urine volume. The median intake based on urinary iodine excretion was above both the EAR and RDA, whereas the value estimated from the food diaries was above the
EAR but below the RDA. The proportion with iodine intake below the EAR (14 and 28\% for intake extrapolated from urine and food diary estimates, respectively) suggested a degree of deficiency within the cohort. However, it is important to acknowledge that because of day-to-day variability in iodine intake, this does not necessarily mean that these individuals were iodine deficient.

The results of the present study highlight the fact that the degree of iodine deficiency in the cohort varies according to the method used for classification. It is important to highlight the fact that the WHO cut-off value for iodine adequacy in adults is based on the fact that goitre risk is low when the median urinary iodine excretion is above $100 \mu \mathrm{g} / \mathrm{d}$, a value that was later used as the cut-off value for a spot-urine sample on the basis that the units (i.e. $\mu \mathrm{g} / \mathrm{d}$ and $\mu \mathrm{g} / \mathrm{l}$ ) are interchangeable ${ }^{(22)}$. If the average urine volume is 1 litre/d,

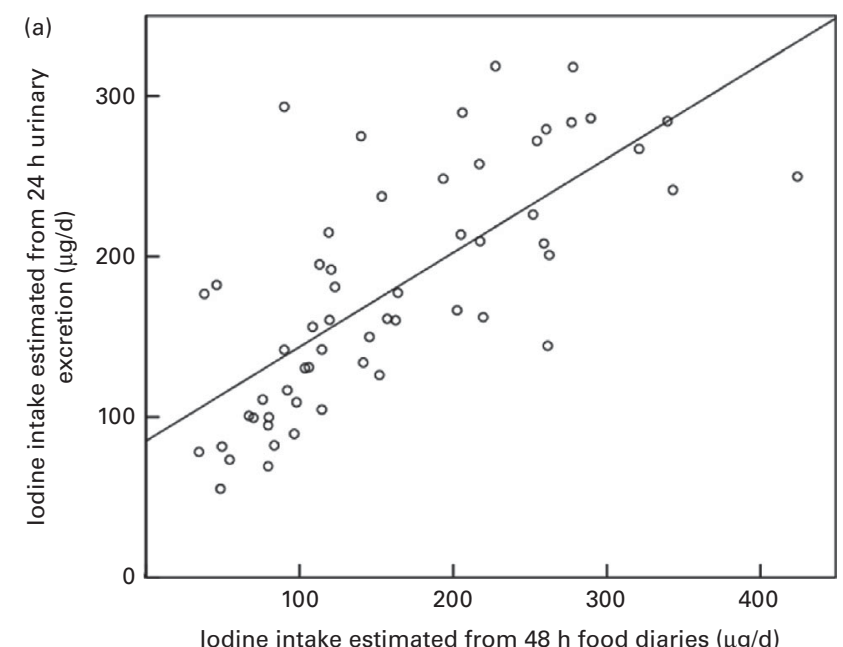

(b)

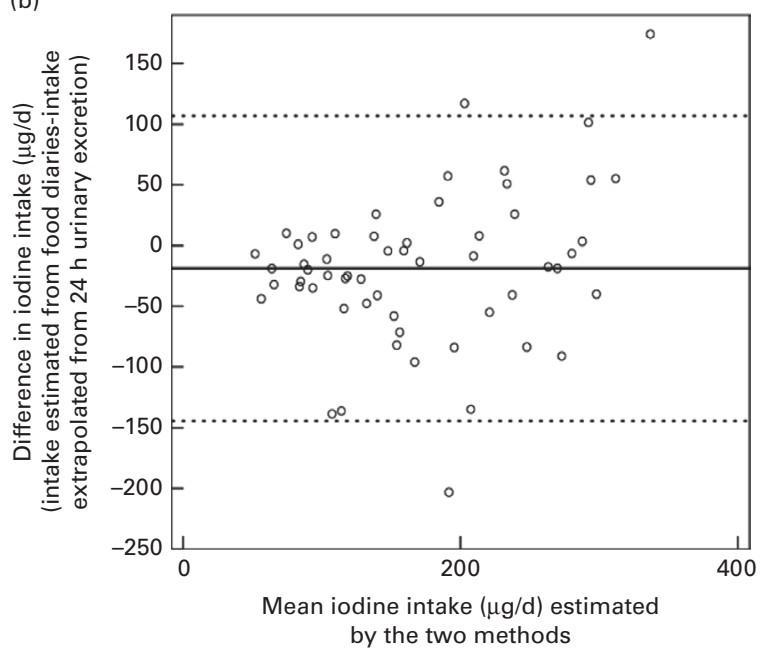

Fig. 2. (a) Correlation between iodine intake estimated from the food diaries and supplements (average of two $24 \mathrm{~h}$ periods) and that extrapolated from $24 \mathrm{~h}$ urinary iodine excretion; $r 0.71\left(r^{2} 0.50\right)$. (b) Bland-Altman plot showing differences between the two methods; - represents the mean difference $(-18.8 \mu \mathrm{g} / \mathrm{d})$ between the two methods and -.- represent the limits of agreement corresponding to $\pm 2 \mathrm{SD}$ (upper limit: $106.8 \mu \mathrm{g} / \mathrm{d}$; lower limit: $-144.4 \mu \mathrm{g} / \mathrm{d})$. 
Table 4. Correlation between dietary components and $24 \mathrm{~h}$ urinary iodine excretion

\begin{tabular}{lccc}
\hline & \multicolumn{3}{c}{$\begin{array}{c}\text { Correlation with 24h urinary } \\
\text { iodine excretion }\end{array}$} \\
\cline { 2 - 4 } Food groups $(\mathrm{g} / \mathrm{d})^{*}$ & $r$ & $P$ & $r^{2}$ \\
\hline Milk & 0.67 & $<0.0001$ & 0.449 \\
Other dairy products & 0.29 & 0.03 & 0.084 \\
Fish & 0.24 & 0.08 & 0.058 \\
Eggs & 0.36 & 0.006 & 0.130 \\
Meat & -0.26 & 0.05 & 0.068 \\
Soya drinks & -0.33 & 0.01 & 0.109 \\
\hline
\end{tabular}

*Average from the $48 \mathrm{~h}$ food diaries $(24 \mathrm{~h}$ before and during the $24 \mathrm{~h}$ of urine collection).

as it is likely to be in children, the units can be used interchangeably, but this is probably not appropriate for adults. Indeed, a lower cut-off value for iodine adequacy in adults has recently been proposed on the basis that the average urine volume in adults is more likely to be 1.5 litres/ $\mathrm{d}$ and thus the cut-off value should be lowered to $60-70 \mu \mathrm{g} / \mathrm{l}^{(22)}$. In fact, in the present study, the mean urine volume was close to 2.5 litres, and this explains why the risk of deficiency is overestimated when using the UIC values rather than the $24 \mathrm{~h}$ urinary iodine excretion values. The food diaries indicated that the participants (mostly nutrition students) drank water throughout the day, and this accounted for the high urine volume observed in the present study. The results of the present study support the need for method-specific $(24 \mathrm{~h}$ $v$. spot collection) and age-specific (adults $v$. schoolchildren/ teenagers) criteria for iodine deficiency ${ }^{(21,22)}$.

On balance, it is likely that this group had a minimal risk of iodine deficiency. Though the median UIC was suggestive of mild deficiency, this is likely to be a result of the high urine volume in the group and therefore dilute urine samples. It is important to point out that these women are by no means representative of UK women of childbearing age as they were highly educated (mostly science degree students/ graduates) in an affluent area of the UK (Surrey). Furthermore, as over $90 \%$ were studying for a degree in nutrition or nutrition/dietetics, it is likely that their knowledge of good nutrition may have skewed the results (see limitations for further explanation).

Although this was a study in women of reproductive age and not in those who were pregnant, there are implications for the pregnant state as iodine intake recommendations are higher for pregnant adults than for non-pregnant adults ${ }^{(7)}$. When intake is extrapolated from the measured $24 \mathrm{~h}$ urinary iodine excretion, $42 \%$ of the participants (or $60 \%$ if using data from the food diaries) did not achieve the EAR for pregnancy (i.e. $160 \mu \mathrm{g} / \mathrm{d}$ ) ${ }^{(7)}$, suggesting that UK women may be unable to meet the increased iodine needs during pregnancy, as previously found in other UK studies ${ }^{(10,13,14)}$ and in recent European studies ${ }^{(5,25-27)}$. Bearing in mind the fact that pregnant women are not given advice on iodine intake ${ }^{(28)}$, they are unlikely to modify their diet to increase the intake of iodine-rich foods when they become pregnant. Indeed, results from the Southampton Women's Survey, where dietary intake was estimated before and after pregnancy in the same women, show that dietary patterns do not change considerably when women become pregnant ${ }^{(29)}$; in terms of iodine-rich foods, the intake of fish and milk does not appear to change in early pregnancy, a time point that is critical for iodine supply for brain development ${ }^{(1,2)}$. The results of the present study suggest that advice to women planning a pregnancy and those who are pregnant should include specific mention of iodine.

\section{Relationship between the two methods used for estimating iodine intake}

The present study provides the first opportunity to evaluate how iodine intake estimated from food diaries compares with iodine intake estimated by extrapolation from $24 \mathrm{~h}$ urinary iodine excretion; the results show a strong correlation between the two methods, both for intake in the $24 \mathrm{~h}$ before urine collection and for intake during the $24 \mathrm{~h}$ of urine collection. This suggests that intake over at least $48 \mathrm{~h}$ contributes to iodine excreted in the $24 \mathrm{~h}$ urine sample, a finding that echoes that of an earlier study carried out in Denmark ${ }^{(30)}$. However, this finding may also be a result of the fact that there was no significant difference in iodine intake between the two $24 \mathrm{~h}$ periods. Despite strong correlations, the Bland-Altman plot showed that iodine intake estimated from the food diaries is lower than that extrapolated from $24 \mathrm{~h}$ urinary iodine excretion (Fig. 2(b)) by approximately $19 \mu \mathrm{g} / \mathrm{d}$, on average; this explains why a higher percentage of participants had iodine intake below the EAR when using the food diaries than when estimating intake from $24 \mathrm{~h}$ urinary iodine excretion (28.1 v. 14\%). This finding is in contrast to data from Denmark, where $24 \mathrm{~h}$ urinary iodine excretion is lower than the intake estimated from either a FFQ or a weighed food diary ${ }^{(31)}$. Food diary analysis has been suggested to be an inaccurate method for estimating iodine intake, in part attributed to the fact that it is difficult to capture the amount of iodine ingested from iodised salt ${ }^{(22,32)}$; however, this criticism is less relevant in the UK where use of iodised salt is low ${ }^{(33,34)}$. The lower iodine intake estimated from food diary analysis in the present study may at least partly be explained by under-reporting - a known problem when using this methodology $^{(35)}$. Furthermore, the food table values for iodine in the WinDiets programme may be inaccurate (as a result of poor-quality or out-of-date iodine data in food composition tables ${ }^{(22)}$ ) and values are missing for certain foods, which may result in an estimate that is lower than the actual intake.

\section{Effect of food consumption on iodine intake and status}

The intake of milk, eggs and dairy products was found to be positively associated with iodine status in the regression analysis. Iodine excretion correlated more strongly with the intake of milk than with that of other dietary components, reflecting the importance of milk and milk products as sources of dietary iodine in the $\mathrm{UK}^{(36)}$ and supporting previous associations between milk intake and urinary iodine status in UK women $^{(12,13)}$. Milk has also been found to be an important source of iodine for adults in other European countries ${ }^{(37-39)}$. 
Interestingly, there was a negative correlation between soyadrink intake and iodine excretion, but intake of soya drink was not a significant predictor of iodine status in a regression model when other dietary sources of iodine were included. This probably reflects the negative correlation between soya drinks and cows' milk intake, suggesting that the negative correlation in univariate analyses was a result of the displacement of iodine-rich cows' milk from the diet. Although the number of soya-drink consumers was relatively low in the present study, our findings warrant further investigation in view of the increasing use of alternatives to cows' milk by UK women; for example, the volume of soya drinks sold in the UK increased by 10.1\% between January 2009 and January $2013^{(40)}$. Very few of these milk alternatives are fortified with iodine and therefore women who rely on these products are likely to be considerably more at risk of iodine deficiency than those who regularly consume cows' milk.

Fish intake was positively correlated with iodine excretion, but the correlation failed to reach significance and was not a significant predictor of iodine status in the regression analysis, perhaps because of the relatively small number of fish consumers in the study. Other $\mathrm{UK}^{(12,13)}$ and European studies ${ }^{(41,42)}$ have failed to find significant associations between iodine status and fish consumption. Egg consumption was positively associated with iodine status as has been found in previous studies in children ${ }^{(43)}$, though not in the study of UK teenage girls $^{(12)}$. The results of the latter study were derived from ambiguous questions on egg consumption, which probably explains the disparity ${ }^{(44)}$.

\section{Study limitations}

The present study is limited by the small number of participants involved; caution should therefore be exercised when interpreting the results of the subgroup analysis of dietary intake (e.g. of soya drinks). Although we had detailed information on the study participants (e.g. $48 \mathrm{~h}$ recorded dietary intake), accuracy would have been improved if we had had a repeated urine collection, even if only for a subsample of the cohort ${ }^{(22,45)}$; we could then have corrected for intra-individual variation in iodine intake. This might have resulted in an improved estimate of intake for individuals falling below the EAR; our estimate of individual intake on the basis of urinary iodine excretion may have resulted in misclassification of the percentage with estimated intake below the EAR. However, at the time that the present study was designed (2006), the use of multiple $24 \mathrm{~h}$ urine collections (as opposed to a single $24 \mathrm{~h}$ collection) was not considered as important as it is now. Completeness of the $24 \mathrm{~h}$ urine sample was self-reported and thus incomplete samples may have been measured; we consider that this is fairly unlikely as the participants were motivated individuals who understood the implications of incomplete urine collections. Finally, the food-diary analysis is limited by the inherent limitations of dietary-intake assessment, including underreporting of intake and use of inaccurate food table values for iodine ${ }^{(31,35)}$. We tried to reduce inaccuracies as far as possible, for example, by using the same researcher to code all diaries and enter the data into WinDiets.

Other limiting factors are that the study was carried out under circumstances likely to have maximised iodine intake and status. First, the sampling was conducted during the winter months and it is known that winter milk has a higher iodine concentration than summer milk due to an increased use of supplemented cattle feed ${ }^{(46-49)}$. Were the study to be repeated in the summer months, the percentage of women classified as iodine deficient would likely be higher. Second, the majority of the participants were students on a nutrition/dietetics degree programme and likely to have had a greater understanding of a healthy diet; indeed, the food diaries demonstrated that the group ate regular meals, with healthy food choices (such as fruit and vegetables) and were perhaps not typical of a population of young UK women. This may have resulted in a relatively high intake of iodine-rich foods such as fish and milk; indeed the average milk intake was higher than that of adult women reported in the recent National Diet and Nutrition Survey (NDNS) $(150 v .124 \mathrm{~g} / \mathrm{d})^{(50)}$.

\section{Conclusion}

For many years, the UK has been assumed to be iodine sufficient, but the present study adds to the growing evidence base that this may not be the case in women of childbearing age. Women entering pregnancy need to have an adequate iodine status to ensure optimal fetal neurological development and pregnancy outcome. The results of the present study suggest that a proportion of UK women may be entering pregnancy with low iodine stores, particularly in view of the fact that the study design probably resulted in a best-case scenario. Further study in UK women of childbearing age is required; from 2015, results will be available on iodine concentration measured in spot-urine samples in the NDNS, which will provide important data on these women. On the basis of the results of the present study, we suggest that urine samples should be corrected for urine dilution (i.e. by measurement of urinary creatinine concentrations). Finally, the present study highlights the need for revised cut-off values for iodine adequacy in adults, given that we found that the classification of iodine status differed depending on whether UIC values or $24 \mathrm{~h}$ urinary iodine excretion measures were used.

\section{Acknowledgements}

The authors are grateful to all study participants and to Dr Christine Sieniawska, at the Trace Element Laboratory in Southampton Hospital, for advice on urinary iodine analysis.

The costs of laboratory analysis and consumables were covered by the University of Surrey. S. C. B. is in receipt of an MRC Population Health Scientist Fellowship, which supported the data analysis and the writing of the manuscript.

The authors contributions' are as follows: S. C. B., M. L. S. and M. P. R. designed the study; S. C. B. and M. L. S. recruited the subjects and participated in the laboratory analysis; S. C. B. conducted the statistical analysis and wrote the manuscript; M. M. analysed the food diaries with supervision from S. C. B.; 
A. W. and A. T. developed the laboratory analysis method and conducted the urine sample analysis; M. P. R. had primary responsibility for the final content. All authors prepared and reviewed the manuscript and approved the final content.

None of the authors has any conflicts of interest to declare.

\section{References}

1. Zimmermann MB (2009) Iodine deficiency. Endocr Rev 30, 376-408.

2. Bath SC, Steer CD, Golding J, et al. (2013) Effect of inadequate iodine status in UK pregnant women on cognitive outcomes in their children: results from the Avon Longitudinal Study of Parents and Children (ALSPAC). Lancet 382, 331-337.

3. Hynes KL, Otahal P, Hay I, et al. (2013) Mild iodine deficiency during pregnancy is associated with reduced educational outcomes in the offspring: 9-year follow-up of the gestational iodine cohort. J Clin Endocrinol Metab 98 , $1954-1962$.

4. Glinoer D (2007) The importance of iodine nutrition during pregnancy. Public Health Nutr 10, 1542-1546.

5. Moleti M, Di Bella B, Giorgianni G, et al. (2011) Maternal thyroid function in different conditions of iodine nutrition in pregnant women exposed to mild-moderate iodine deficiency: an observational study. Clin Endocrinol (Oxf) 74, 762-768.

6. Moleti M, Lo Presti VP, Campolo MC, et al. (2008) Iodine prophylaxis using iodized salt and risk of maternal thyroid failure in conditions of mild iodine deficiency. J Clin Endocrinol Metab 93, 2616-2621.

7. Food and Nutrition Board \& Institute of Medicine (2001) Dietary Reference Intakes for Vitamin A, Vitamin K, Arsenic, Boron, Chromium, Copper, Iodine, Manganese, Molybdenum, Nickel, Silicon, Vanadium and Zinc. Washington, DC: National Academy Press.

8. Phillips DI (1997) Iodine, milk, and the elimination of endemic goitre in Britain: the story of an accidental public health triumph. J Epidemiol Community Health 51, 391-393.

9. de Benoist B, McLean E, Andersson M, et al. (2008) Iodine deficiency in 2007: global progress since 2003. Food Nutr Bull 29, 195-202.

10. Kibirige MS, Hutchison S, Owen CJ, et al. (2004) Prevalence of maternal dietary iodine insufficiency in the north east of England: implications for the fetus. Arch Dis Child Fetal Neonatal Ed 89, F436-F439.

11. Barnett C, Visser T, Williams F, et al. (2002) Inadequate iodine intake of $40 \%$ of pregnant women from a region in Scotland. J Endocrinol Invest 25, Suppl. 7, 90.

12. Vanderpump MP, Lazarus JH, Smyth PP, et al. (2011) Iodine status of UK schoolgirls: a cross-sectional survey. Lancet 377, 2007-2012.

13. Bath SC, Walter A, Taylor A, et al. (2014) Iodine deficiency in pregnant women living in the South East of the UK: the influence of diet and nutritional supplements on iodine status. Br J Nutr 111, 1622-1631.

14. Pearce EN, Lazarus JH, Smyth PP, et al. (2010) Perchlorate and thiocyanate exposure and thyroid function in first-trimester pregnant women. J Clin Endocrinol Metab 95, 3207-3215.

15. Thomson CD, Colls AJ, Conaglen JV, et al. (1997) Iodine status of New Zealand residents as assessed by urinary iodide excretion and thyroid hormones. BrJ Nutr $\mathbf{7 8}, 901-912$.
16. Vejbjerg P, Knudsen N, Perrild H, et al. (2009) Estimation of iodine intake from various urinary iodine measurements in population studies. Thyroid 19, 1281-1286.

17. WHO, UNICEF \& ICCIDD (2007) Assessment of Iodine Deficiency Disorders and Monitoring their Elimination, 3rd ed. Geneva: World Health Organisation.

18. Konig F, Andersson M, Hotz K, et al. (2011) Ten repeat collections for urinary iodine from spot samples or 24-hour samples are needed to reliably estimate individual iodine status in women. J Nutr 141, 2049-2054.

19. Caldwell KL, Makhmudov A, Jones RL, et al. (2005) EQUIP: a worldwide program to ensure the quality of urinary iodine procedures. Accred Qual Assur 10, 356-361.

20. Food Standards Agency (2002) Food Portion Sizes, 3rd ed. London: The Stationary Office.

21. Als C, Minder C, Willems D, et al. (2003) Quantification of urinary iodine: a need for revised thresholds. Eur J Clin Nutr 57, 1181-1188.

22. Zimmermann MB \& Andersson M (2012) Assessment of iodine nutrition in populations: past, present, and future. Nutr Rev 70, 553-570.

23. Department of Health (1991) Report on Health and Social Subjects: Volume 41. Dietary Reference Values for Food, Energy and Nutrients for the United Kingdom. London: The Stationery Office.

24. Beaton GH (2006) When is an individual an individual versus a member of a group? an issue in the application of the dietary reference intakes. Nutr Rev 64, 211-225.

25. Vandevijvere S, Amsalkhir S, Mourri AB, et al. (2013) Iodine deficiency among Belgian pregnant women not fully corrected by iodine-containing multivitamins: a national cross-sectional survey. Br J Nutr 109, 2276-2284.

26. Aguayo A, Grau G, Vela A, et al. (2013) Urinary iodine and thyroid function in a population of healthy pregnant women in the North of Spain. J Trace Elem Med Biol 27, 302-306.

27. Raverot V, Bournaud C, Sassolas G, et al. (2012) Pregnant French women in the Lyon area are iodine deficient and have elevated serum thyroglobulin concentrations. Thyroid 22, 522-528.

28. NHS Choices (2011) Your health during pregnancy. Vitamins, minerals and special diets. In The Pregnancy Care Planner. http://www.nhs.uk/Planners/pregnancycare planner/pages/Vitaminsmineralsdiets.aspx (accessed 19 July 2011)

29. Crozier SR, Robinson SM, Godfrey KM, et al. (2009) Women's dietary patterns change little from before to during pregnancy. J Nutr 139, 1956-1963.

30. Rasmussen LB, Ovesen L \& Christiansen E (1999) Day-to-day and within-day variation in urinary iodine excretion. Eur J Clin Nutr 53, 401-407.

31. Rasmussen LB, Ovesen L, Bulow I, et al. (2002) Dietary iodine intake and urinary iodine excretion in a Danish population: effect of geography, supplements and food choice. BrJ Nutr 87, 61-69.

32. Rasmussen LB, Ovesen L, Bulow I, et al. (2001) Evaluation of a semi-quantitative food frequency questionnaire to estimate iodine intake. Eur J Clin Nutr 55, 287-292.

33. Bath S, Button S \& Rayman MP (2014) Availability of iodised table salt in the UK - is it likely to influence population iodine intake? Public Health Nutr 17, 450-454.

34. Lazarus JH \& Smyth PP (2008) Iodine deficiency in the UK and Ireland. Lancet 372, 888.

35. Livingstone MB, Prentice AM, Strain JJ, et al. (1990) Accuracy of weighed dietary records in studies of diet and health. BMJ 300, 708-712. 
36. Henderson L, Irving K, Gregory J, et al. (2003) The National Diet E Nutrition Survey: Adults Aged 19 to 64 Years. Volume 3: Vitamin and Mineral Intake and Urinary Analytes. London: HMSO.

37. Gunnarsdottir I, Gustavsdottir AG, Steingrimsdottir L, et al. (2013) Iodine status of pregnant women in a population changing from high to lower fish and milk consumption. Public Health Nutr 16, 325-329.

38. Rasmussen LB, Carle A, Jorgensen T, et al. (2008) Iodine intake before and after mandatory iodization in Denmark: results from the Danish Investigation of Iodine Intake and Thyroid Diseases (DanThyr) study. Br J Nutr 100, 166-173.

39. Soriguer F, Garcia-Fuentes E, Gutierrez-Repiso C, et al. (2012) Iodine intake in the adult population. Di@bet.es study. Clin Nutr 31, 882-888.

40. Datum DC (2014) Kantar Worldpanel Liquid - Soya. http:// www.dairyco.org.uk/resources-library/market-information/ dairy-sales-consumption/kantar-worldpanel-liquid-milkmarket/ (accessed 10 March 2014).

41. Brantsaeter AL, Haugen M, Thomassen Y, et al. (2010) Exploration of biomarkers for total fish intake in pregnant Norwegian women. Public Health Nutr 13, 54-62.

42. Johner SA, Thamm M, Nothlings U, et al. (2013) Iodine status in preschool children and evaluation of major dietary iodine sources: a German experience. Eur J Nutr 52, 1711-1719.

43. Remer T, Fonteyn N, Alexy U, et al. (2006) Longitudinal examination of 24-h urinary iodine excretion in schoolchildren as a sensitive, hydration status-independent research tool for studying iodine status. Am J Clin Nutr 83, 639-646.

44. Bath S \& Rayman MP (2011) Iodine deficiency in UK schoolgirls. Lancet 378, 1623, author reply 1624 .

45. Charlton KE, Batterham MJ, Buchanan LM, et al. (2014) Intraindividual variation in urinary iodine concentrations: effect of adjustment on population distribution using two and three repeated spot urine collections. BMJ Open $\mathbf{4}$, e003799.

46. Phillips DI, Nelson M, Barker DJ, et al. (1988) Iodine in milk and the incidence of thyrotoxicosis in England. Clin Endocrinol (Oxf) 28, 61-66.

47. Lee SM, Lewis J, Buss DH, et al. (1994) Iodine in British foods and diets. Br J Nutr 72, 435-446.

48. Wenlock RW, Buss DH, Moxon RE, et al. (1982) Trace nutrients. 4. Iodine in British food. Br J Nutr 47, 381-390.

49. Food Standards Agency (2008) Retail survey of iodine in UK produced dairy foods. FSIS 02/08. http://www.food. gov.uk/multimedia/pdfs/fsis0208.pdf (accessed 11 October 2010).

50. Bates B, Lennox A, Prentice A, et al. (2012) NDNS Headline Results from Years 1, 2 and 3 (Combined) of the Rolling Programme (2008/2009-2010/2011). London: Food Standards Agency/Department of Health. http://media.dh.gov.uk/ network/261/files/2012/07/NDNS-Y3-report_All-TEXT-docscombined.pdf (accessed 29 May 2013). 